\title{
YOUNG GALAXY CANDIDATES IN THE HUBBLE FRONTIER FIELDS. I. A2744
}

\author{
Wei Zheng ${ }^{1}$, Xinwen Shu ${ }^{2,3}$, John Moustakas ${ }^{4}$, Adi Zitrin ${ }^{5,15}$, Holland C. Ford ${ }^{1}$, XingXing Huang ${ }^{1,3}$, \\ Tom Broadhurst ${ }^{6,7}$, Alberto Molino ${ }^{8}$, Jose M. Diego ${ }^{9}$, Leopoldo Infante ${ }^{10}$, Franz E. Bauer ${ }^{10,11,12}$, \\ Daniel D. Kelson ${ }^{13}$, and RensKe Smit ${ }^{14}$ \\ ${ }^{1}$ Department of Physics and Astronomy, Johns Hopkins University, Baltimore, MD 21218, USA \\ ${ }^{2}$ CEA Saclay, DSM/Irfu/Service d'Astrophysique, Orme des Merisiers, F-91191 Gif-sur-Yvette Cedex, France \\ ${ }^{3}$ Department of Astronomy, University of Science and Technology of China, Hefei, Anhui 230026, China \\ ${ }^{4}$ Department of Physics and Astronomy, Siena College, Loudonville, NY 12211, USA \\ ${ }^{5}$ Cahill Center for Astronomy and Astrophysics, California Institute of Technology, MS 249-17, Pasadena, CA 91125, USA \\ ${ }^{6}$ Department of Theoretical Physics, University of Basque Country UPV/EHU, Bilbao, Spain \\ ${ }^{7}$ IKERBASQUE, Basque Foundation for Science, E-48013 Bilbao, Spain \\ ${ }^{8}$ Instituto de Astrofísica de Andalucía-CSIC, Glorieta de la Astronomía, s/n. E-18008 Granada, Spain \\ ${ }^{9}$ IFCA, Instituto de Física de Cantabria, UC-CSIC, s/n. E-39005 Santander, Spain \\ ${ }^{10}$ Instituto de Astrofísica, Facultad de Física, Pontificia Universidad Católica de Chile 306, Santiago 22, Chile \\ ${ }^{11}$ Millennium Institute of Astrophysics, Vicuña Mackenna 4860, 7820436 Macul, Santiago, Chile \\ ${ }^{12}$ Space Science Institute, Boulder, CO 80301, USA \\ ${ }^{13}$ The Observatories of the Carnegie Institution for Science, Pasadena, CA 91101, USA \\ ${ }^{14}$ Leiden Observatory, Leiden University, NL-2300 RA Leiden, The Netherlands \\ Received 2014 February 25; accepted 2014 September 10; published 2014 October 15
}

\begin{abstract}
We report the discovery of 24 Lyman-break candidates at $7 \lesssim z \lesssim 10.5$, in the Hubble Frontier Fields (HFF) imaging data of A2744 $(z=0.308)$, plus Spitzer/IRAC data and archival ACS data. The sample includes a triple image system with a photometric redshift of $z \simeq 7.4$. This high redshift is geometrically confirmed by our lens model corresponding to deflection angles that are $12 \%$ larger than the lower-redshift systems used to calibrate the lens model at $z=2.019$. The majority of our high-redshift candidates are not expected to be multiply lensed given their locations in the image plane and the brightness of foreground galaxies, but are magnified by factors of $\sim 1.3-15$, so that we are seeing further down the luminosity function than comparable deep-field imaging. It is apparent that the redshift distribution of these sources does not smoothly extend over the full redshift range accessible at $z<12$, but appears to break above $z=9$. Nine candidates are clustered within a small region of $20^{\prime \prime}$ across, representing a potentially unprecedented concentration. Given the poor statistics, however, we must await similar constraints from the additional HFF clusters to properly examine this trend. The physical properties of our candidates are examined using the range of lens models developed for the HFF program by various groups including our own, for a better estimate of underlying systematics. Our spectral-energy-distribution fits for the brightest objects suggest stellar masses of $\simeq 10^{9} M_{\odot}$, star formation rates of $\simeq 4 M_{\odot} \mathrm{yr}^{-1}$, and a typical formation redshift of $z \lesssim 19$
\end{abstract}

Key words: cosmology: observations - galaxies: clusters: individual (A2744) - galaxies: high-redshift gravitational lensing: strong

Online-only material: color figures

\section{INTRODUCTION}

Our understanding of the first billion years of cosmic time has increased significantly in recent years, thanks to the Hubble Space Telescope's (HST's) Wide-Field Camera 3/Infrared Channel (WFC3/IR; Kimble et al. 2008) as well as the Spitzer Space Telescope's Infrared Array Camera (IRAC; Fazio et al. 2004). Until recently, the Hubble Ultra Deep Field (Beckwith et al. 2006; Illingworth et al. 2013) has provided our deepest view of the universe, revealing a considerable number of galaxy candidates at $z>7$, including one candidate at $z \simeq 10$ (Bouwens et al. 2011, 2012; Ellis et al. 2013; Oesch et al. 2013; Illingworth et al. 2013). The cosmic epoch of $z \simeq 10$ is important to study as it marks the dawn of galaxy formation and the beginning of reionization of the intergalactic medium. However, galaxies at that redshift are extremely faint, making it difficult to discover and study the abundant population of galaxies below $L^{*}$, the knee of the luminosity function. Fortunately, the

\footnotetext{
${ }^{15}$ Hubble Fellow.
}

magnification boost afforded by gravitational lensing combined with HST's exquisite imaging capabilities in the near-infrared (NIR), provides an avenue for both discovering and characterizing the intrinsic properties of galaxies around $z \simeq 12$, when the universe was less than half a billion years old.

The Cluster Lensing And Supernova survey with Hubble (CLASH; Postman et al. 2012) carried out HST imaging of 25 galaxy clusters in 16 broad bands between $0.2-1.7 \mu \mathrm{m}$ to a depth of $A B$ magnitude $\sim 27$ with a total of 20 orbits per cluster. The CLASH program has led to many interesting discoveries of magnified, intrinsically faint galaxies. Several hundred dropout galaxies have been uncovered in the range $z \simeq 6-8$, with a few notable examples at higher redshifts of $z \simeq 9-11$ (see Zheng et al. 2012b; Bouwens et al. 2014a; Coe et al. 2013; Bradley et al. 2014), helping to motivate dedicated deeper lensing surveys.

The Hubble Frontier Fields (HFF) is a new initiative now being carried out to observe the distant universe to an unprecedented depth, combining the power of deep HST imaging and gravitational lensing. In HST's Cycles 21 and 22, 560 orbits of Director's Discretionary Time have been allocated to 
Table 1

Summary of Observations

\begin{tabular}{lllcc}
\hline \hline Telescope & Band & \multicolumn{1}{c}{ Date } & $\begin{array}{c}\text { Exposure Time } \\
(\mathrm{s})\end{array}$ & $\begin{array}{c}\text { Limiting } \\
\text { Magnitude (5 } \sigma)\end{array}$ \\
\hline$H S T$ & F160W & 2013 Aug-2014 Jul & 71664 & 28.7 \\
$H S T$ & F140W & 2013 Oct and Nov & 28140 & 28.5 \\
$H S T$ & F125W & 2013 Oct-2014 Jul & 36620 & 28.6 \\
$H S T$ & F105W & 2013 Aug-2014 Jul & 71209 & 29.0 \\
$H S T$ & F814W & 2014 May-Jul, 2009 Oct & 117518 & 29.4 \\
$H S T$ & F606W & 2014 May, 2009 Oct & 36882 & 29.2 \\
$H S T$ & F435W & 2014 Jun-Jul, 2009 Oct & 61909 & 29.3 \\
Spitzer & IRAC1 & 2013 Sep, 2014 Jan and Feb & 339291 & 25.3 \\
Spitzer & 2004 Jun and Nov & 339291 & \\
& IRAC2 & 2013 Sep, 2014 Jan and Feb & & \\
\hline
\end{tabular}

Table 2

WFC3 and ACS Photometry ${ }^{\mathrm{a}}$ of Candidates at $z>9$

\begin{tabular}{|c|c|c|c|c|c|c|c|c|c|}
\hline Name & $\begin{array}{l}\text { Photometric } \\
\text { Redshift }\end{array}$ & $\begin{array}{l}\text { R.A. } \\
\text { (J2000) }\end{array}$ & $\begin{array}{l}\text { Decl. } \\
\text { (J2000) }\end{array}$ & F160W & F140W & F125W & F105W & F814W & $\mu^{\mathrm{b}}$ \\
\hline $\mathrm{JD} 2^{\mathrm{c}}$ & $10.5 \pm 0.7$ & 3.597037 & -30.412132 & $28.00 \pm 0.15$ & $29.00 \pm 0.43$ & $30.20 \pm 1.33$ & $29.52 \pm 0.52$ & $>31.00$ & $14.8_{-7.8}^{+66}$ \\
\hline JD3 & $10.0_{-1.0}^{+0.7}$ & 3.603344 & -30.390978 & $28.68 \pm 0.16$ & $29.26 \pm 0.30$ & $30.30 \pm 0.78$ & $>30.50$ & $>30.50$ & $1.6_{-0.1}^{+0.9}$ \\
\hline
\end{tabular}

Notes.

${ }^{\text {a }}$ Magnitudes are isophotal, scaled by an aperture correction term derived in the F160W band. The errors and limiting magnitudes are $1 \sigma$. Photometric redshifts have been derived using BPZ, and the quoted uncertainties indicate the $68 \%$ confidence interval.

b Magnification factor from ZITRIN "NFW" model (see Section 4.1), where uncertainties are combined from two sources: (1) the model statistical uncertainties, which are relatively small; and more prominently, (2) the maximum differences with other six models, excluding one highest and one lowest values.

${ }^{\mathrm{c}}$ For system JD1, see Zitrin et al. (2014).

Table 3

WFC 3 and ACS Photometry ${ }^{\mathrm{a}}$ of Candidates at $8 \lesssim z<9$

\begin{tabular}{|c|c|c|c|c|c|c|c|c|c|}
\hline Name & $\begin{array}{l}\text { Photometric } \\
\text { Redshift }\end{array}$ & $\begin{array}{l}\text { R.A. } \\
\text { (J2000) }\end{array}$ & $\begin{array}{l}\text { Decl. } \\
\text { (J2000) }\end{array}$ & F160W & F140W & F125W & F105W & F814W & $\mu^{\mathrm{b}}$ \\
\hline YD1 & $8.7_{-0.2}^{+0.5}$ & 3.603856 & -30.381905 & $27.83 \pm 0.08$ & $27.86 \pm 0.09$ & $28.49 \pm 0.17$ & $29.85 \pm 0.43$ & $>31.00$ & $1.4_{-0.1}^{+0.7}$ \\
\hline YD2 & $8.3 \pm 0.2$ & 3.572515 & -30.413267 & $28.12 \pm 0.12$ & $28.11 \pm 0.13$ & $28.11 \pm 0.13$ & $29.84 \pm 0.48$ & $>31.00$ & $1.6_{-0.1}^{+0.4}$ \\
\hline YD3 & $8.8_{-0.2}^{+0.4}$ & 3.603858 & -30.415842 & $28.33 \pm 0.10$ & $28.63 \pm 0.15$ & $28.66 \pm 0.18$ & $>30.50$ & $>31.00$ & $2.9_{-0.6}^{+0.5}$ \\
\hline $\mathrm{YD}^{\mathrm{c}}$ & $8.5 \pm 0.1$ & 3.603864 & -30.382265 & $26.42 \pm 0.04$ & $26.46 \pm 0.04$ & $26.82 \pm 0.06$ & $28.78 \pm 0.27$ & $>31.00$ & $1.4_{-0.1}^{+0.7}$ \\
\hline YD5 & $8.5 \pm 0.3$ & 3.579479 & -30.386534 & $27.83 \pm 0.10$ & $27.66 \pm 0.09$ & $28.38 \pm 0.18$ & $29.24 \pm 0.29$ & $>31.00$ & $3.0_{-0.9}^{+9.8}$ \\
\hline YD8 $^{c}$ & $8.1 \pm 0.1$ & 3.596096 & -30.385832 & $26.65 \pm 0.04$ & $26.49 \pm 0.04$ & $26.68 \pm 0.04$ & $27.76 \pm 0.09$ & $>31.00$ & $1.9_{-0.2}^{+5.6}$ \\
\hline YD9 & $8.3_{-0.6}^{+0.2}$ & 3.572902 & -30.413658 & $28.49 \pm 0.14$ & $28.22 \pm 0.12$ & $28.68 \pm 0.18$ & $29.62 \pm 0.32$ & $>31.00$ & $1.6_{-0.1}^{+0.5}$ \\
\hline YD10 & $8.3 \pm 0.2$ & 3.598108 & -30.382393 & $27.70 \pm 0.09$ & $27.57 \pm 0.09$ & $27.89 \pm 0.12$ & $28.89 \pm 0.23$ & $>31.00$ & $1.5_{-0.1}^{+2.3}$ \\
\hline YD11 & $8.0_{-0.6}^{+0.3}$ & 3.600947 & -30.399149 & $28.96 \pm 0.17$ & $28.97 \pm 0.19$ & $28.89 \pm 0.18$ & $>30.50$ & $>31.00$ & $3.4_{-0.1}^{+0.9}$ \\
\hline
\end{tabular}

Notes.

a Magnitudes are isophotal, scaled by an aperture correction term derived in the F160W band. The errors and limiting magnitudes are $1 \sigma$. Photometric redshifts have been derived using BPZ, and the quoted uncertainties indicate the $68 \%$ confidence interval.

b Magnification factor from ZITRIN "NFW" model (see Section 4.1), where uncertainties are combined from two sources: (1) the model statistical uncertainties, which are relatively small; and more prominently, (2) the maximum differences with other six models, excluding one highest and one lowest values.

${ }^{c}$ Also in Coe et al. (2014).

observe four clusters. The observations are carried out with four WFC3/IR filters (F160W, F140W, F125W, F105W) and three ACS filters (Advanced Camera for Surveys; Ford et al. 1998, F814W, F606W, F435W). It is anticipated that 280 orbits will be allocated in Cycle 23 to observe two additional clusters. In addition, deep Spitzer and Chandra observations are planned for the six HFF fields. These coordinated observations will enable us to probe the star formation rate density at $z \gtrsim 9$, study the faint end of the galaxy population at $z \simeq 3-8$, and map the dark matter in these clusters in unprece- dented detail via many multiple images of background sources (Hubble Deep Fields Initiative 2012 Science Working Group Report). ${ }^{16}$

We report the discovery of 24 candidate Lyman-break galaxies (LBGs) at $z \gtrsim 7$ in the field of A2744, based on the HFF observations and archival data (Table 1). The faintest sources detected are around AB magnitude 29. These objects, listed in

\footnotetext{
16 http://www.stsci.edu/hst/campaigns/frontier-fields/documents/HDFI_ SWGReport2012.pdf
} 
Table 4

WFC3 and ACS Photometry ${ }^{\mathrm{a}}$ of Candidates at $7 \lesssim z<8$

\begin{tabular}{|c|c|c|c|c|c|c|c|c|c|}
\hline Name & $\begin{array}{c}\text { Photometric } \\
\text { Redshift }\end{array}$ & $\begin{array}{c}\text { R.A. } \\
(\mathrm{J} 2000)\end{array}$ & $\begin{array}{c}\text { Decl. } \\
(\mathrm{J} 2000)\end{array}$ & F160W & F140W & F125W & F105W & F814W & $\mu^{\mathrm{b}}$ \\
\hline ZD1 & $7.4_{-0.6}^{+0.3}$ & 3.603582 & -30.382442 & $28.52 \pm 0.19$ & $28.09 \pm 0.14$ & $28.47 \pm 0.21$ & $>30.50$ & $>31.00$ & $1.4_{-0.1}^{+0.7}$ \\
\hline $\mathrm{ZD} 2^{\mathrm{c}, \mathrm{d}}$ & $7.9 \pm 0.1$ & 3.604520 & -30.380472 & $25.56 \pm 0.03$ & $25.73 \pm 0.03$ & $25.91 \pm 0.04$ & $26.83 \pm 0.07$ & $>30.00$ & $1.3_{-0.1}^{+0.7}$ \\
\hline $\mathrm{ZD}^{\mathrm{c}, \mathrm{d}}$ & $7.7 \pm 0.1$ & 3.606477 & -30.380993 & $26.45 \pm 0.04$ & $26.57 \pm 0.05$ & $26.64 \pm 0.05$ & $27.50 \pm 0.08$ & $>31.00$ & $1.3_{-0.1}^{+1.0}$ \\
\hline ZD4 & $7.8 \pm 0.3$ & 3.605263 & -30.380606 & $27.97 \pm 0.12$ & $27.95 \pm 0.13$ & $28.29 \pm 0.17$ & $29.05 \pm 0.26$ & $>31.00$ & $1.3_{-0.1}^{+0.7}$ \\
\hline $\mathrm{ZD}^{\mathrm{d}}$ & $7.6_{-0.3}^{+0.1}$ & 3.588985 & -30.378662 & $27.55 \pm 0.05$ & $27.77 \pm 0.07$ & $27.52 \pm 0.06$ & $28.29 \pm 0.09$ & $>30.00$ & $1.6_{-0.1}^{+0.9}$ \\
\hline $\mathrm{ZD}^{\mathrm{c}}$ & $7.5 \pm 0.1$ & 3.606575 & -30.380928 & $26.38 \pm 0.04$ & $26.45 \pm 0.04$ & $26.86 \pm 0.06$ & $27.39 \pm 0.08$ & $>31.00$ & $1.3_{-0.1}^{+1.1}$ \\
\hline ZD7 & $7.3 \pm 0.2$ & 3.592285 & -30.409911 & $26.98 \pm 0.06$ & $26.99 \pm 0.07$ & $26.92 \pm 0.06$ & $27.39 \pm 0.07$ & $>30.50$ & $5.9_{-3.0}^{+7.8}$ \\
\hline ZD8 & $7.5 \pm 0.3$ & 3.579668 & -30.398678 & $28.33 \pm 0.12$ & $28.11 \pm 0.11$ & $28.29 \pm 0.14$ & $28.89 \pm 0.17$ & $>31.00$ & $14.0_{-6.8}^{+38}$ \\
\hline $\mathrm{ZD} 9^{\mathrm{c}}$ & $7.0 \pm 0.1$ & 3.603208 & -30.410368 & $26.48 \pm 0.04$ & $26.68 \pm 0.06$ & $26.71 \pm 0.06$ & $26.83 \pm 0.05$ & $>31.00$ & $3.4_{-0.8}^{+7.8}$ \\
\hline ZD10 & $7.0 \pm 0.3$ & 3.581282 & -30.404207 & $28.75 \pm 0.16$ & $28.61 \pm 0.16$ & $28.78 \pm 0.19$ & $28.98 \pm 0.17$ & $>31.00$ & $9.8_{-4.4}^{+15}$ \\
\hline $\mathrm{ZD} 11^{\mathrm{c}}$ & $7.0 \pm 0.1$ & 3.585321 & -30.397964 & $27.49 \pm 0.04$ & $27.45 \pm 0.04$ & $27.33 \pm 0.04$ & $27.55 \pm 0.03$ & $>31.00$ & $4.7_{-3.1}^{+1.8}$ \\
\hline
\end{tabular}

Notes.

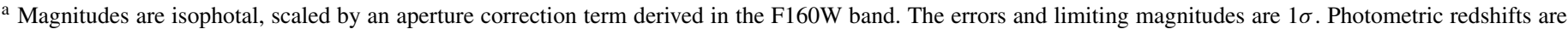
BPZ with $1 \sigma$ error.

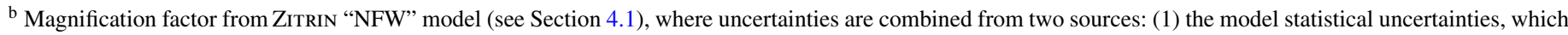
are relatively small; and more prominently, (2) the maximum differences with other six models, excluding one highest and one lowest values.

c Reported by A14 and Laporte et al. (2014).

d Also in Coe et al. (2014).

Tables 2-4, have "secure" photometric redshifts greater than 7 and a negligible probability $(<1 \%)$ of being at lower redshift.

We adopt a concordance cosmology with $\Omega_{M}=0.3, \Omega_{\Lambda}=$ 0.7 , and $h=H_{0} / 100 \mathrm{~km} \mathrm{~s}^{-1} \mathrm{Mpc}^{-1}=0.7$, and the $\mathrm{AB}$ magnitude system throughout.

\section{DATA}

A2744 $(z=0.308)$ is the first HFF target in HST's Cycle 21 . It is one of the most actively merging galaxy clusters known (Merten et al. 2011), displaying a large critical curve of roughly $60^{\prime \prime} \times 30^{\prime \prime}$. The six HFF clusters have been selected to maximize the lensing boost, which means that systems with highly complex mass distributions (e.g., clusters in the process of merging) have been selected (Torri et al. 2004; Redlich et al. 2012; Zitrin et al. 2013b). The HFF observations of A2744 (GO/DD 13495, PI: Lotz) were carried out between 2013 October 25 and 2014 July 1 . Additional WFC3/IR images obtained in 2013 August and 2014 June-July (GO 13386, PI: Rodney) and ACS images of 2009 (GO 11689, PI: Dupke) are retrieved from the Mikulski Archive for Space Telescopes $\left(\mathrm{MAST}^{17}\right)$ and used. Table 1 lists the exposure times and limiting magnitudes for all the imaging used in our analysis.

We process the $H S T$ data using APLUS (Zheng et al. 2012a), an automated pipeline modified from the APSIS package (Blakeslee et al. 2003) with an enhanced capability of processing WFC3 data and aligning them with the ACS data. We retrieve the calibrated images from the $H S T$ instrument pipelines, namely the $f l c$ images for ACS (corrected for the detector charge transfer efficiency) and $f t$ images for WFC3/IR. Recently, we have updated APLUS so that images of individual exposures are aligned using DrizzlePac (Hack et al. 2013), achieving an astrometric precision of $\sim 0 . \prime 015$ or better. Figure 1 displays a composite color image of the cluster field.

Using APLUS, we align, resample, and combine all the available imaging in each filter to a common pixel scale of

\footnotetext{
${ }^{17}$ http://archive.stsci.edu/hst
}

0'065, which is half of WFC3/IR's pixel scale and slightly larger than that of ACS. We then create detection images from the inverse-variance weighted sum of the WFC3/IR and ACS images, respectively, and run SExtractor (Bertin \& Arnouts 1996) in dual-image mode. The $z \gtrsim 7$ candidates are first selected from the NIR catalog derived from the WFC3/IR detection image, with a threshold of 1.5 times the signal-tonoise value over a minimum of 4 pixels. We choose colors measured from isophotal magnitudes to select our high-redshift candidates (see Section 3), as they balance the need between depth and photometric precision (Ferguson \& McGaugh 1995). The $5 \sigma$ limiting magnitude in the WFC3/IR bands is $\sim 28.8$ in a 0'4 diameter aperture (see Table 1), and $\sim 29.2$ for the observed-frame optical ACS bands.

As part of the HFF campaign, deep Spitzer/IRAC images were obtained in 2013 September and 2014 January and February in Channels 1 and 2 at wavelengths 3.1-3.9 and 3.9-5.0 $\mu \mathrm{m}$, respectively, using Director's Discretionary Time (Program 90257, PI: Soifer). The effective exposure time in each channel, including that of the archival data (Program 84; PI: Rieke) obtained in 2004, is $\sim 339$ ks. The IRAC corrected Basic Calibrated Data (cBCD) images are processed with MOPEX (Makovoz \& Khan 2005) and sampled to a final pixel scale of 0.6. In order to perform background matching of the individual cBCD frames we run all steps in the Overlap module, where we use the SExtractor background estimation with a window size of 25 pixels. To create the mosaic images we run all three outlier modules (i.e. Dual outlier, Mosaic Outlier, and Box Outlier) and we use the default interpolation, with the fine resolution parameter $=0$. The estimated $1 \sigma$ limiting magnitude is 27.3 for IRAC channel 1 (IRAC1, $3.6 \mu \mathrm{m}$ ) and 27.1 for channel 2 (IRAC2, $4.5 \mu \mathrm{m}$; see Table 1).

\section{SELECTION}

We search for LBGs using their distinct color around $0.1216(1+z) \mu \mathrm{m}$. For example, at $z \simeq 7-8$, the Lyman break is 


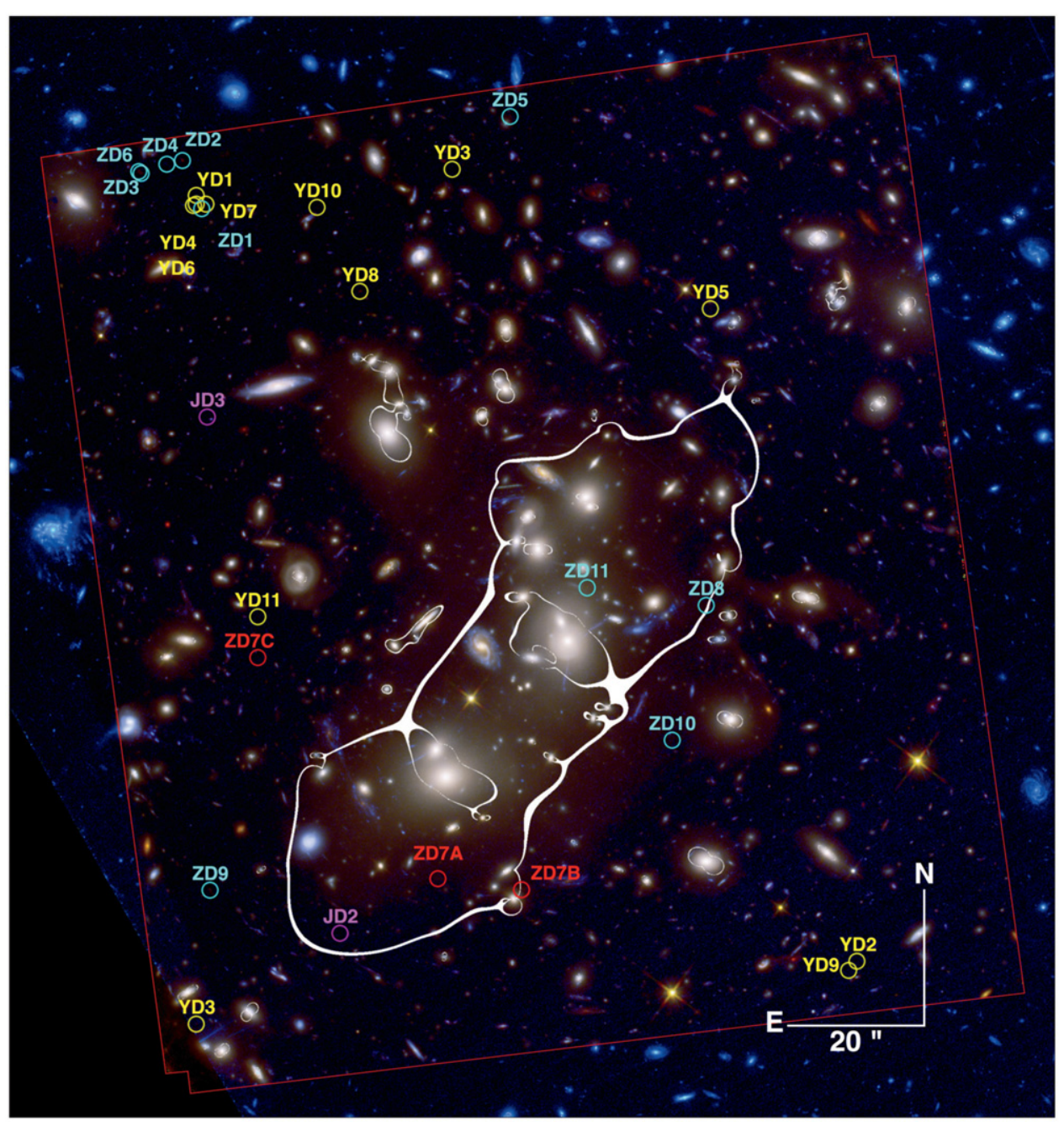

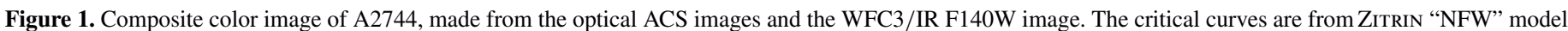

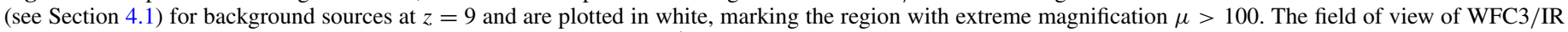

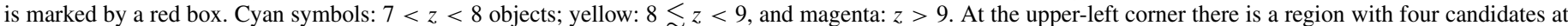
$z \simeq 7.5$ and five at $z \simeq 8.5$. In the lower-left part a triple system of $z \simeq 7.4$ is marked in red.

(A color version of this figure is available in the online journal.)

at $\sim 1 \mu \mathrm{m}$, between the F814W and F125W bands. Our selection criteria, in units of magnitude, are as follows:

$\mathrm{F} 814 \mathrm{~W}-\mathrm{F} 105 \mathrm{~W}>0.8$

$\mathrm{F} 105 \mathrm{~W}-\mathrm{F} 125 \mathrm{~W}<0.6$

$\mathrm{F} 814 \mathrm{~W}-\mathrm{F} 105 \mathrm{~W}>0.8+(\mathrm{F} 105 \mathrm{~W}-\mathrm{F} 125 \mathrm{~W})$.

These color cuts are similar to those utilized in previous work such as Oesch et al. (2010).

For $z \simeq 8-9$, the break is at $\sim 1.15 \mu \mathrm{m}$, between the $\mathrm{F} 105 \mathrm{~W}$ and F140W bands:

$\mathrm{F} 105 \mathrm{~W}-\mathrm{F} 140 \mathrm{~W}>0.8$

F140W $-\mathrm{F} 160 \mathrm{~W}<0.6$

$\mathrm{F} 105 \mathrm{~W}-\mathrm{F} 140 \mathrm{~W}>0.8+(\mathrm{F} 140 \mathrm{~W}-\mathrm{F} 160 \mathrm{~W})$.

For $z \simeq 10$, the break is between the F125W and F160W bands: F125W - F160W >0.8.

We require that a candidate must not be detected above $1 \sigma$ in a summed image blueward of the selection bands defined above. For objects at $z \simeq 7$, this requires a non-detection in a summed image of the F606W and F435W bands, while for candidates at $z \gtrsim 8$ this requires a non-detection in the stacked optical detection image.

In addition to the color selection criteria described above, we also exclude candidates lying within 1 arcsec of the detector edges, in order to mitigate potentially spurious photometry. We also exclude candidates lying near stellar diffraction spikes, which are difficult to remove because HFF WFC3/IR exposures were obtained at the same position angle. Finally, we also identify and remove candidates with a color decrement of F160W - IRAC > 3, as they are most likely extremely red objects at lower redshift $(z \simeq 2)$.

Our HST photometry is measured within an isophotal aperture, but aperture-corrected to a total flux using the mag_auto mag_iso difference in the F160W band. In a few cases where source blending is significant, we visually inspect the images and choose an aperture that is larger than the source's FWHM, but not so large as to be affected by nearby sources, and use the corresponding aperture magnitude in place of mag_auto. We also verify that our aperture colors (and therefore our list of highredshift candidates) are not affected by image artifacts if we use the publicly released HST mosaics based on the Mosaicdrizzle pipeline (Koekemoer et al. 2013). ${ }^{18}$

The IRAC images of our candidates suffer from crowding due to the instrument's large point spread function (PSF, FWHM $\simeq$ 1 ".6), such that simple aperture photometry might result in inaccurate fluxes due to contamination from nearby sources.

\footnotetext{
18 http://archive.stsci.edu/pub/hlsp/frontier/abell2744/images/hst/v1.0
} 
Table 5

IRAC Photometry for Selected Candidates ${ }^{\mathrm{a}}$

\begin{tabular}{lcc}
\hline \hline Name & IRAC 1 & IRAC 2 \\
\hline JD2 & $>27.3$ & $>27.1$ \\
YD1 & $>27.3$ & $26.2 \pm 0.5$ \\
YD2 & $>27.3$ & $>27.1$ \\
YD4 $^{\mathrm{b}}$ & $25.8 \pm 0.3$ & $25.4 \pm 0.2$ \\
YD6 & $25.5 \pm 0.2$ & $25.2 \pm 0.2$ \\
YD7 $^{\mathrm{b}}$ & $26.5 \pm 0.6$ & $26.2 \pm 0.5$ \\
YD8 & $26.5 \pm 0.5$ & $>27.1$ \\
YD9 & $>27.3$ & $>27.1$ \\
YD10 & $>27.3$ & $>27.1$ \\
YD11 & $>27.3$ & $>27.1$ \\
ZD1 & $25.7 \pm 1.3$ & $26.1 \pm 1.4$ \\
ZD2 & $26.6 \pm 0.7$ & $25.0 \pm 0.2$ \\
ZD3 & $>27$ & $26.1 \pm 0.5$ \\
ZD4 & $26.5 \pm 0.6$ & $>27.1$ \\
ZD6 $^{b}$ & $26.0 \pm 0.4$ & $25.7 \pm 0.4$ \\
ZD9 $^{\mathrm{b}}$ & $26.6 \pm 0.4$ & $26.6 \pm 0.3$ \\
\hline
\end{tabular}

Notes.

a IRAC photometry of eight other candidates is not practical because of heavy contaminations from nearby sources.

b Two respective close pairs within one IRAC pixel, each fitted as one component. The fluxes of individual components are partitioned by a ratio of their fluxes in the F160W band.

To address this issue, we use a deblending technique whereby contaminating neighbors are subtracted using GALFIT (Peng et al. 2010) by performing a fit to the objects of interest and all their close neighbors simultaneously in a $\sim 10^{\prime \prime} \times 10^{\prime \prime}$ fitting window around the source of interest (Overzier et al. 2009; Zheng et al. 2012b). The IRAC PSF is determined from the same image by stacking 10 bright, isolated point sources. Positions and radial profiles of neighboring sources in this region are derived from the higher resolution HST F160W-band mosaic, while the initial input magnitudes are obtained by running SExtractor on the IRAC images. During the fitting process, all input parameters are allowed to vary, except for the positions of the objects of interest. This process is similar to that of Labbé et al. (2006) and Labbé et al. (2010). Eight of our candidates are so heavily blended by nearby bright sources with complex radial profiles that GALFIT fails to satisfactorily model contaminating emission from bright sources, leading to an over- or undersubtraction of fluxes and hence a large $\chi^{2}$ value for the fit. As a result, the fluxes for these eight candidates are not measurable with reasonable uncertainties. Our analysis yields a total of 16 sources for which photometry or upper limits from GALFIT are possible (see Table 5).

We carry out extensive tests to check the reliability of our IRAC photometry for each source. First, we place a simulated point source of magnitude 25 near the candidate and run GALFIT with different fitting windows and background levels until the expected magnitude of each simulated source is recovered (with a magnitude difference $<0.1 \mathrm{mag}$ compared to the input value, consistent with the photometric errors). We then proceed to fit the flux of each candidate, using the fitting window and background level on the image that recovered the brightness of the simulated source. We repeat these tests at three different positions for the simulated source to verify our measurement of the source magnitude. To account for the uncertainties in estimating the background, we choose the average magnitude of three measurements as the source magnitude, which are reported in Table 5.

In Figures 2-4 we show cutout images of all the candidates.

\section{MODELS}

\subsection{Gravitational Lensing Models}

As part of the HFF initiative, seven independently derived gravitational lensing models of the A2744 field were developed and publicly released through the MAST archive. ${ }^{19}$ For our analysis we adopt the ZITRIN "NFW" model as our fiducial lensing model, and utilize the other six models to help quantify the systematic uncertainties in our magnification estimates. The ZITRIN "NFW" model assumes a pseudo-isothermal elliptical mass distribution for each cluster galaxy, scaled by the galaxy luminosity, and an elliptical NFW (Navarro et al. 1996) halo for the dark matter. In the case of A2744, two such elliptical NFW halos are used, centered on the two central, brightest cluster galaxies to represent the global dark-matter component. These are combined with the galaxies component to generate, via a long Monte-Carlo-Markov-Chain minimization, the bestfitting model for the total projected mass (see Zitrin et al. 2009, 2013a, 2013b, and references therein).

To estimate the systematic uncertainty in the magnification of each of our high-redshift candidates, we exclude the highest and lowest magnification factors and then calculate the difference in the second-highest and second-lowest magnifications from the different models. This procedure is designed to mitigate potential extremes in the model predictions, and better reflect the true systematic uncertainties. The magnification factors listed in Tables 2-4 are the best-fit values at the tabulated redshift based on the ZITRIN "NFW" model, while the uncertainties are the quadrature sum of the systematic and statistical uncertainties. Figure 1 shows the composite color image of the A2744 field, overlaid with the critical curves and identification numbers for all our candidates.

\subsection{Photometric Redshifts}

We calculate photometric redshifts using the Bayesian photometric redshift code BPZ (Bayesian Photometric Redshifts; Benítez 2000; Coe et al. 2006), adopting the same template library used by the CLASH collaboration (Jouvel et al. 2014). The template set consists of five elliptical galaxy templates, two spiral galaxy templates, and four starburst galaxy templates with moderately strong emission lines. The templates were originally based on the PÉGASE stellar population synthesis models (Fioc \& Rocca-Volmerange 1997), but have been recalibrated using spectroscopic redshifts of galaxies with deep, multiband photometry from the FIREWORKS survey (Wuyts et al. 2008). We assume ignorant (i.e., flat) priors on both galaxy type and redshift in the range $z=0-12$.

Using BPZ, we identify 24 candidates that satisfy our color selection criteria (see Section 3) and whose photometric redshifts place them at $z>7$. In Tables $2-4$ we list the coordinates, photometric redshifts, HST photometry, and magnifications of these 24 candidates.

In order to infer the physical properties of our high-redshift candidates (see Section 5.2), and as an additional check on the BPZ-based photometric redshifts, we use the Bayesian spectral energy distribution (SED) modeling code iSEDfit (Moustakas

\footnotetext{
19 For details, please see the Acknowledgements and http://archive.stsci.edu/prepds/frontier/lensmodels.
} 


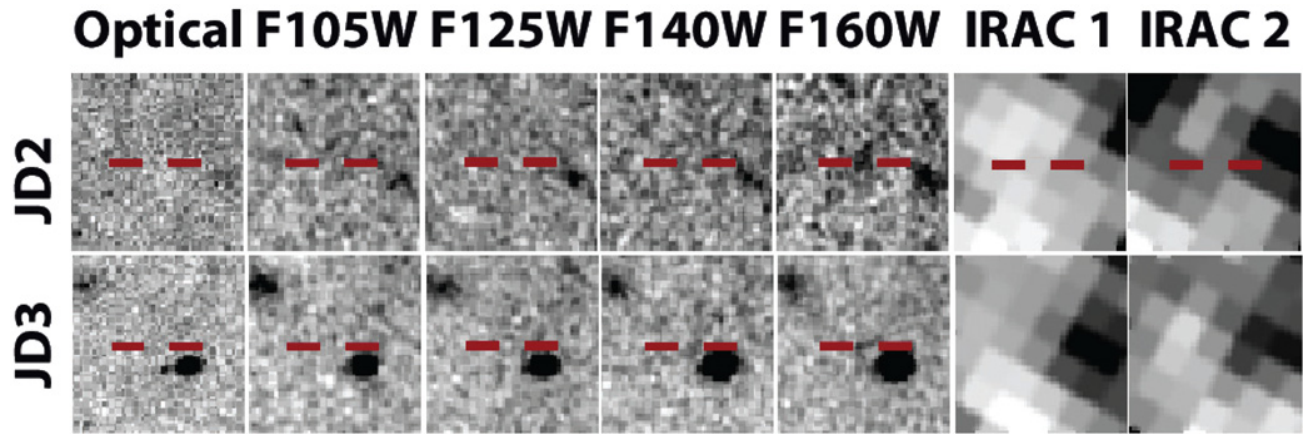

Figure 2. Cutout images of LBG candidates of $z>9$ in A2744. The optical images are from the respective ACS detection images, which are the weighted sums of ACS data in the F814W, F606W, and F435W bands. Each candidate is at the image center, marked by pairs of red bars. For JD3, no red bars are present as no photometry is made because of a bright nearby source. Using the HST photometry of this bright source, we estimate the fluxes at 3.6 and $4.5 \mu \mathrm{m}$ with SED models and find that the best-fit model may account for all the observed IRAC fluxes. Therefore it is likely that source JD3 is weak in the two IRAC bands. The field of view is 3.3 , north is up and east to the left.

(A color version of this figure is available in the online journal.)

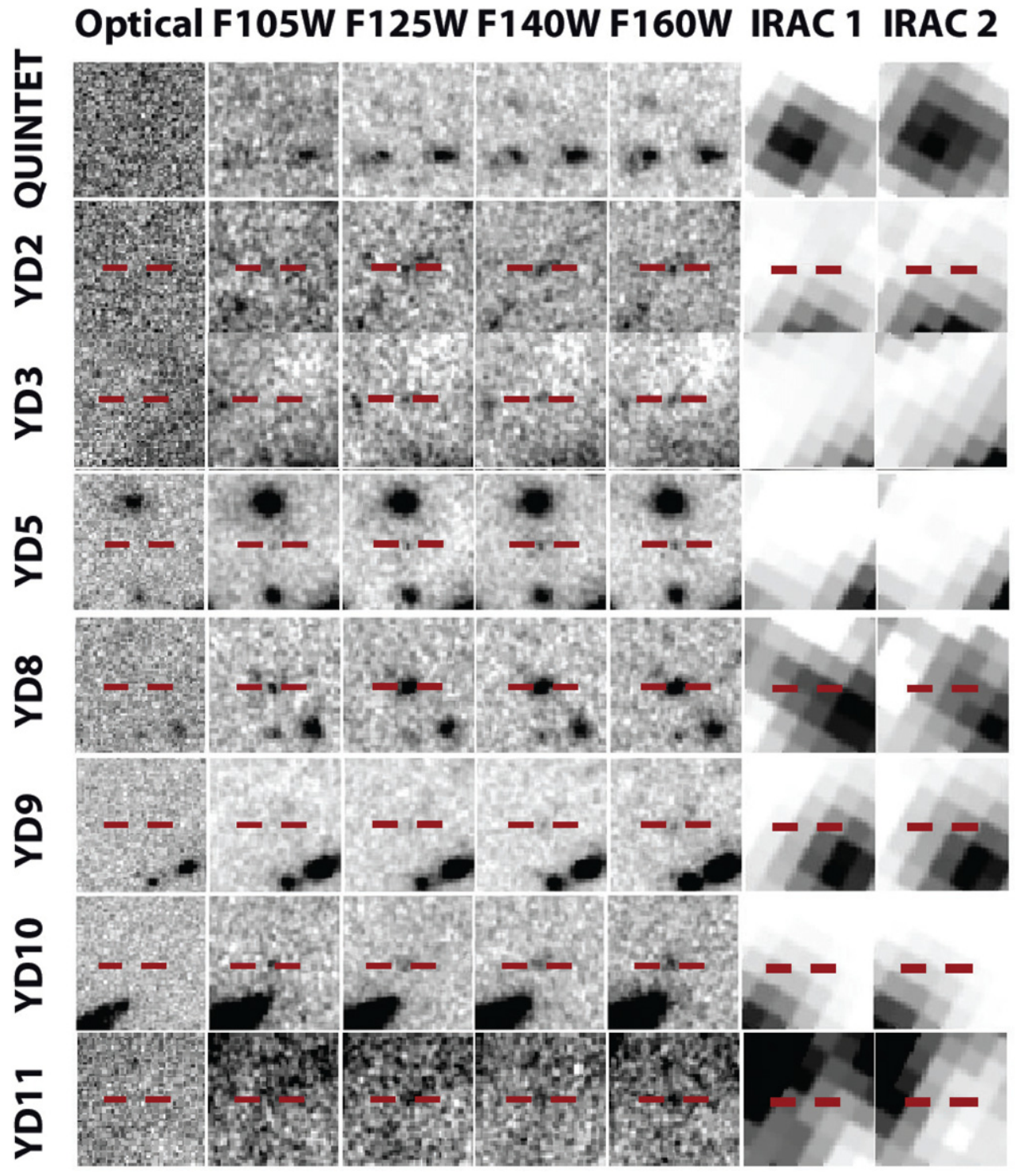

Figure 3. Cutout images of LBG candidates of $8 \lesssim z<9$ in A2744. The symbols are the same as Figure 2. For the "Quintet" field, there are multiple candidates: YD1, YD4, YD6, YD7, and ZD1 (see Figure 7 for identification). For other fields, each candidate is at the image center, marked by pairs of red bars. For YD3 and YD5, no red bars are present as no photometry is made. The field of view is 3 .'3, north is up and east to the left.

(A color version of this figure is available in the online journal.) 


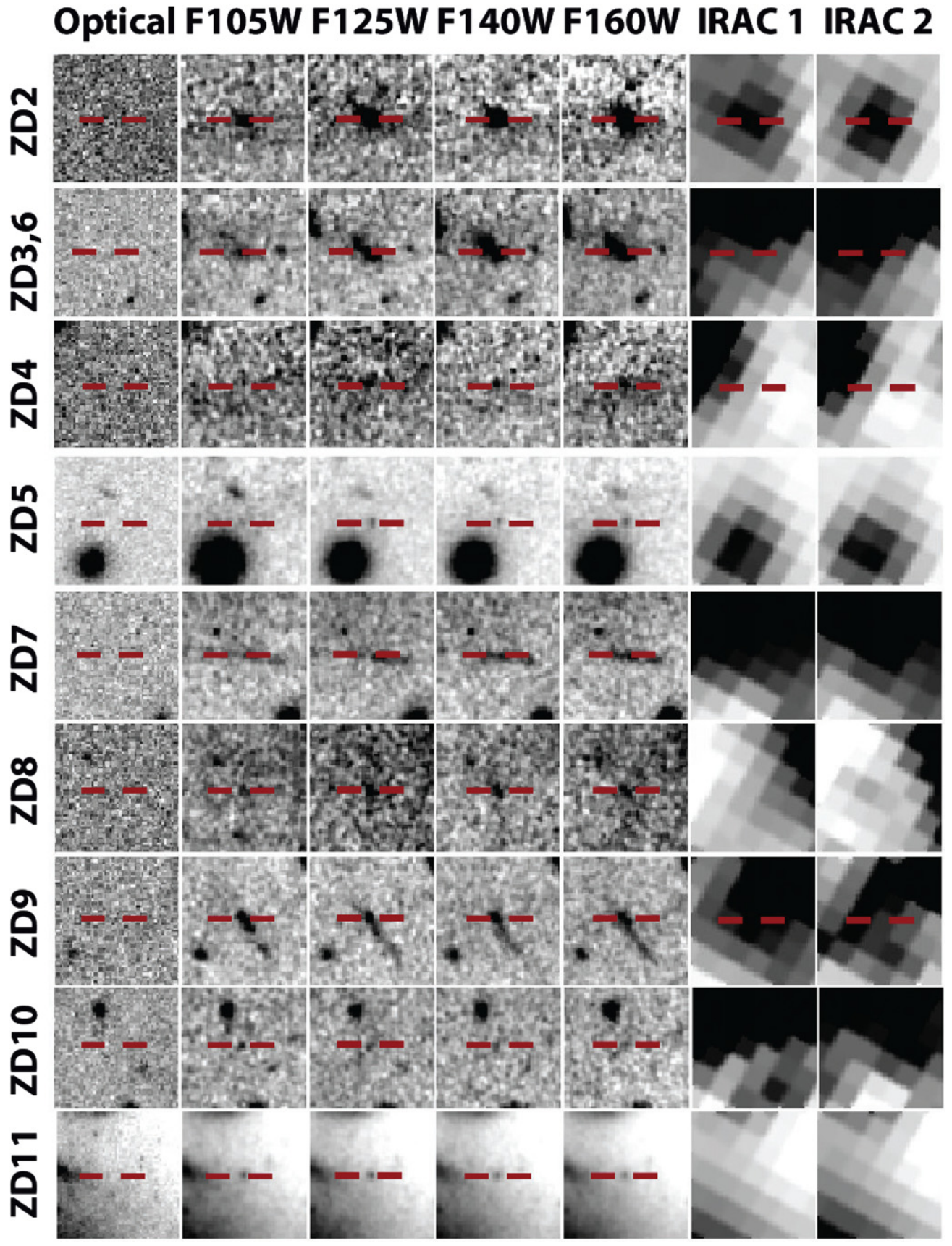

Figure 4. Cutout images of LBG candidates of $7 \lesssim z<8$ in A2744. The symbols are the same as Figure 3 .

(A color version of this figure is available in the online journal.)

et al. 2013). Using a Monte Carlo technique, we generate 20,000 model SEDs with a broad range of star formation histories, ages, stellar metallicities, dust content, and nebular emissionline strength. We use the Flexible Stellar Population Synthesis models (v 2.4; Conroy et al. 2009; Conroy \& Gunn 2010) based on the MILES stellar library (Sanchez-Blázquez et al. 2006) and assume the Chabrier (2003) initial mass function from $0.1-100 M_{\odot}$. We adopt delayed star formation histories, $\operatorname{SFR}(t) \propto t e^{-t / \tau}$, where SFR is the star formation rate, $t$ is the time since the onset of star formation ("age"), and $\tau$ is the characteristic time for star formation. The advantage of this parameterization is that it allows for both linearly rising $(t \ll \tau)$ and exponentially declining $(t \gtrsim \tau)$ star formation histories, which may be important for modeling the SEDs of galaxies at the highest redshifts (e.g., Papovich et al. 2011). For our photometric redshift calculations we adopt uniform priors on age $t \in[0.01,12] \mathrm{Gyr}^{20}{ }^{20}$ star formation timescale $\tau \in[0.01,5.0]$ Gyr, stellar metallicity $Z / Z_{\odot} \in[0.04,1.6]$, and rest-frame $V$-band attenuation $A_{V} \in[0-3] \mathrm{mag}$, assuming the time-dependent attenuation curve of Charlot \& Fall (2000). Each model also includes nebular emission lines whose luminosity is tied self-consistently to the number of hydrogen-ionizing photons.

We find that iSEDfit and BPZ yield statistically consistent photometric redshifts for the majority of the candidates; the ${ }^{20}$ Note that the age of the stellar population is never allowed to be older than
the age of the universe at the redshift under consideration. 

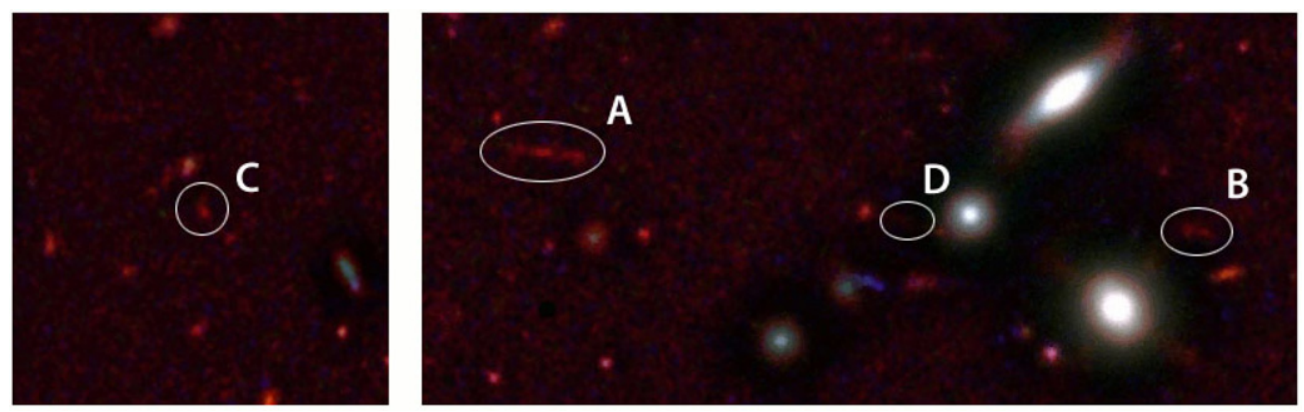

Figure 5. Red arclet ZD7 (marked as A) at redshift 7.4 and its two counter images B and C. Both images have been processed to boost the signal-to-noise of faint red objects and to reduce the contribution from nearby bright galaxies. An extremely faint source, marked as D, may be the potential fourth counter image. (A color version of this figure is available in the online journal.)

Table 6

Photometry ${ }^{\mathrm{a}}$ of Multiple System

\begin{tabular}{|c|c|c|c|c|c|c|c|c|}
\hline Name & $\begin{array}{c}\text { R.A. } \\
(\mathrm{J} 2000)\end{array}$ & $\begin{array}{c}\text { Decl. } \\
(\mathrm{J} 2000)\end{array}$ & F125W & F160W - F125W & F140W - F125W & F105W - F125W & F814W - F125W & $\mu$ \\
\hline ZD7A1 & 3.592410 & -30.409897 & $27.33 \pm 0.08$ & $-0.08 \pm 0.10$ & $-0.05 \pm 0.10$ & $0.38 \pm 0.11$ & $>4.0$ & $5.7_{-2.7}^{+7.5}$ \\
\hline ZD7B & 3.588430 & -30.410340 & $27.7 \pm 0.1$ & $-0.3 \pm 0.1$ & $0.0 \pm 0.1$ & $0.6 \pm 0.3$ & $>2.0$ & $32.5_{-32}^{+31}$ \\
\hline ZD7C & 3.600940 & -30.400824 & $28.58 \pm 0.15$ & $0.25 \pm 0.22$ & $-0.21 \pm 0.22$ & $0.81 \pm 0.28$ & $>2.0$ & $2.8_{-0.1}^{+1.5}$ \\
\hline
\end{tabular}

Note. ${ }^{\text {a }}$ See notes in Table 4.

mean difference is $\Delta z=-0.14 \pm 0.11$ (iSEDfit minus BPZ), which is well within our quoted photometric redshift uncertainties. In a few cases iSEDfit prefers a lower-redshift solution, $z \approx 2$; however, in every case these lower-redshift solutions require a highly unlikely combination of physical properties, namely low stellar masses, low star formation rates, and large amounts of dust attenuation. Secondary peaks in the redshift probability distribution from iSEDfit, on the other hand, place these five candidates at $z>7$, in agreement with BPZ's primary redshift probability peaks.

\subsection{Multiple Systems}

To help corroborate the high-redshift nature of our 24 candidates, we search for potential counter images near the locations predicted by the gravitational lensing model. Among the candidates that are inside or near the $z=7$ critical curves, JD1 (Zitrin et al. 2014) and ZD7 are the only two cases where multiple images are found. For the others, the predicted counter images are either behind bright foreground galaxies, or too faint to be confirmed. Two of the counter images predicted for source ZD10 are near that of ZD11, which have been discussed by A14 (system 5).

The triple system ZD7 is shown in Figure 5 and Table 6. Image $A$ is an arclet made of two components that are separated only by $\sim 1.5 \mathrm{kpc}$ in the source plane, assuming a magnification of six. This is similar to a case in A1689 where a pair of LBGs at $z \simeq 7.6$ may be merging (Bradley et al. 2008). Image $\mathrm{B}$ is behind a bright foreground galaxy, which we subtract before carrying out photometry. We make use of the pure geometric scaling induced by strong lensing to estimate a purely geometric distance for this triply imaged case. The ZITRIN "NFW" lensing model described in Section 4.1 is based on eleven sets of multiply lensed galaxies between $2<z<4$, including a spectroscopic redshift of system 6 at $z=2.019$ (Richard et al. $2014^{21}$ ). This spectroscopic redshift provides a

\footnotetext{
21 http://www.stsci.edu/hst/campaigns/frontier-fields/FF-Data
}

normalization of the model so that the deflection field induced in the lens plane, $\boldsymbol{\alpha}_{\boldsymbol{L}}(\boldsymbol{\theta})$, can be scaled to any redshift via the lensing source distance ratio $f(z)=d_{l s}(z) / d_{s}(z)$ to provide the observed deflection field $\boldsymbol{\alpha}(\boldsymbol{\theta})=d_{l s}(z) / d_{s}(z) \boldsymbol{\alpha}_{\boldsymbol{L}}(\boldsymbol{\theta})$. Hence only a simple scaling of the relative lensing distance ratios is required to relate deflections at any given redshift to the lensing distance of the normalization used to calibrate the lens model, which in our case is $f(z) / f(z=2.019)$. We find that this factor is $\sim 1.12$ for the triple system which minimizes the location of the observed images relative to that generated by the model, and this corresponds to a best BPZ estimate of $z \simeq 7.4$. A possible faint fourth image $\mathrm{D}$ is noted between images $\mathrm{A}$ and $\mathrm{B}$, close to another bright galaxy (Figure 5).

\section{DISCUSSION}

\subsection{Individual Candidates}

Our paper serves as an independent verification of dropout objects in other reports, including the recent work of Atek et al. (2014, A14 hereafter), Laporte et al. (2014), Coe et al. (2014), and Lam et al. (2014). Most of the 15 candidates in A14 are at $6<z<7$, and therefore have only limited overlap with ours. Three of their candidates have been independently identified by us, but with somewhat different photometric redshifts. Object ZD9 in Table 4, with $z_{\text {phot }}=7.0$, corresponds to A14's object $561\left(z_{\mathrm{A} 14}=7.5\right)$. Object ZD2 $\left(z_{\text {phot }}=7.9\right)$ is A14's object 2070 $\left(z_{\mathrm{A} 14}=8.35\right)$, object $\mathrm{Y} 1(z=7.98)$ in Laporte et al. (2014), and object 2493-2561 in Coe et al. (2014). Object ZD11 $\left(z_{\text {phot }}=7.0\right)$ is A14's object $5.2\left(z_{\mathrm{A} 14}=6.4\right)$ and system $17\left(z_{\text {phot }}=6.75\right)$ in Lam et al. (2014). The close pair of ZD3/ZD6 $\left(z_{\text {phot }}=7.7\right)$ are object $2070\left(z_{\mathrm{A} 14}=8.35\right)$ and object 2555-2516 in Coe et al. (2014). Objects YD4, YD7, YD8, YD10, ZD2, ZD3, ZD5 are objects 2493-2561, 2481-2561, 2306-3090, 2355-2566, 25082497, 2555-2516, 2136-2432 in Coe et al. (2014), respectively.

The IRAC2 flux of object ZD2 (Y1 in Laporte et al. 2014) is more than three times the IRAC1 value, which suggests a strong Balmer break. A similar case at $z \simeq 6$ was reported 


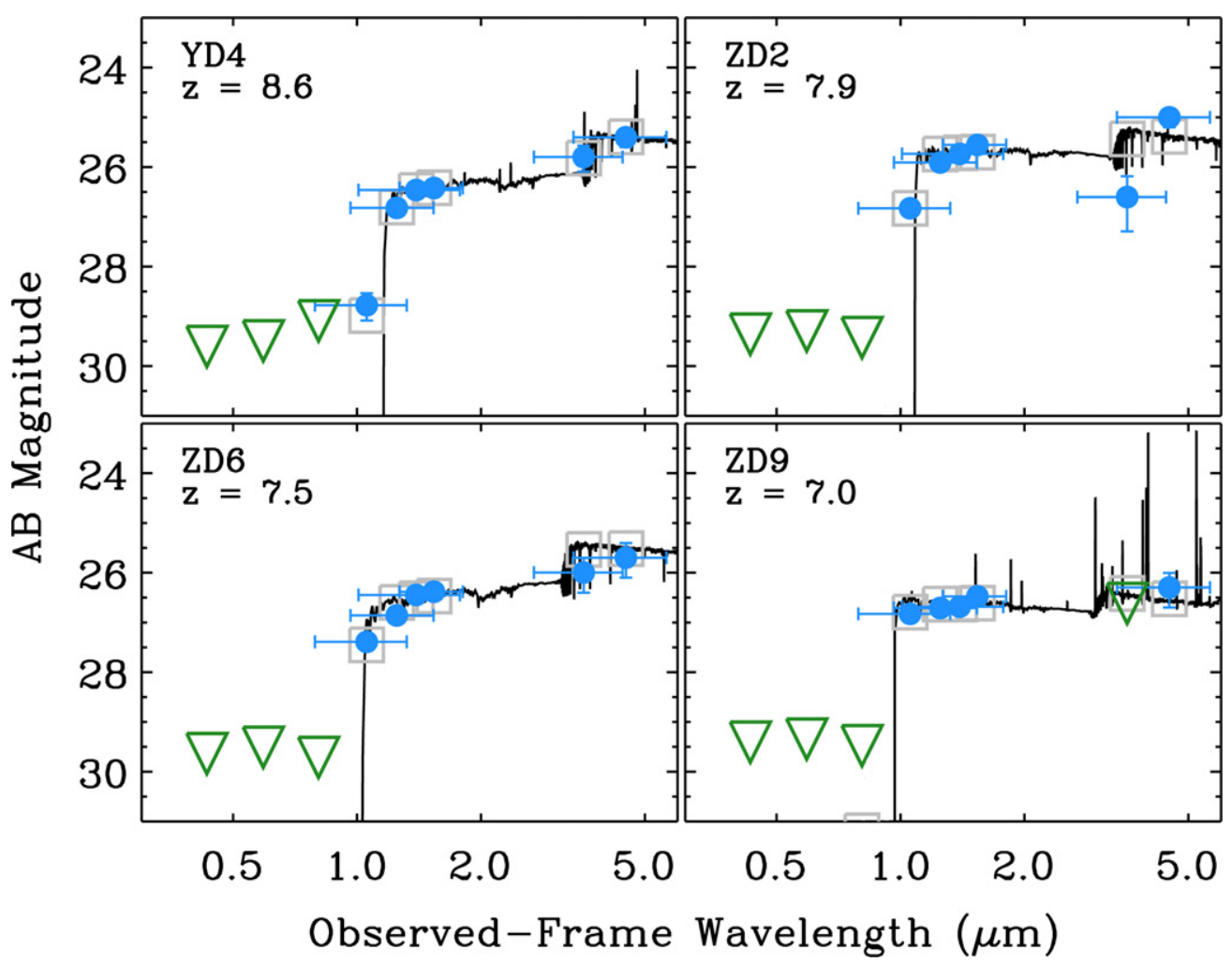

Figure 6. Observed-frame SEDs of four bright candidates that have well-measured IRAC photometry. The filled blue points show the observed photometry, while the open green triangles indicate $2 \sigma$ upper limits. The black spectrum shows the best-fitting (maximum likelihood) SED based on our Bayesian SED modeling using iSEDfit. The large gray squares show the photometry of the best-fitting model convolved with the ACS, WFC3, and IRAC filter response curves.

(A color version of this figure is available in the online journal.)

in A383 (Richard et al. 2011) in which both the IRAC1 and IRAC2 magnitudes are higher than the HST photometry by 1.6 magnitude. At redshift $z \simeq 8$, the Balmer break is in the IRAC1 band, and the model prediction is higher than the measured IRAC1 flux.

\subsection{Physical Properties}

In addition to reporting on the discovery of our highredshift candidates, we can also begin to characterize their physical properties. We defer a more detailed analysis of the full sample to a forthcoming paper. Here, we focus on the 10 objects at $z>7$ with the highest-confidence photometric redshifts which have well-measured photometry in at least one IRAC channel. Photometry redward of the Balmer break is particularly important for placing meaningful constraints on the stellar mass and age of the stellar populations in these distant objects.

To infer the physical properties of these galaxies we use iSEDfit, but adopt a more restricted set of priors than the ones used to estimate photometric redshifts (see Section 4.2). Specifically, we adopt uniform priors on galaxy age $t \in$ $[10,750]$ Myr, $\tau \in[10,1000]$ Myr, stellar metallicity $Z / Z_{\odot} \in$ $[0.04,1.0]$, and we assume no dust attenuation (e.g., Bouwens et al. 2010). Recall that the age of the universe at $z=7-8$ in our adopted concordance cosmology is 750-630 Myr.

We find that our $z>7$ candidates have demagnified stellar masses of around $10^{9} M_{\odot}$, and SFRs of approximately $4 M_{\odot} \mathrm{yr}^{-1}$. These results imply an average doubling time of around $500 \mathrm{Myr}$, which is comparable to the age of the universe at $z \simeq 8 .^{22}$ The ages of the galaxies in our sample are less well constrained given the uncertainties in our IRAC photometry; nevertheless, we find a median SFR-weighted age for the sample of $\lesssim 430$ Myr (95\% confidence interval), corresponding to a typical formation redshift of $z \lesssim 19$. Figure 6 presents the SEDs of four representative galaxies in our sample, sorted by decreasing redshift, as well as the maximum likelihood fits derived using iSEDfit.

\subsection{Source Clustering}

An apparent concentration of candidates northeast of the cluster center is shown in Figure 1 . Nine objects at $z \simeq 7-8$ are found within a region of $20^{\prime \prime}$. Since the average magnification in that area is not high $(\mu \simeq 1.4)$, this apparent overdensity is likely intrinsic rather than being due to lensing. In addition, Figure 7 shows a small region "Quintet" where five candidates are located within approximately $2^{\prime \prime}$ of one another: objects YD1, YD4, YD6, YD7, and ZD1. These objects have similar estimated photometric redshifts of between 7.9 and 8.6, and their projected separations in the source plane are within $\sim 8 \mathrm{kpc}$. Given the uncertainties in our photometric redshifts, it is therefore possible that these sources are physically associated.

To estimate the uncertainties in IRAC photometry for these sources whose separations are close to the IRAC PSF size, we place 500 sets of simulated sources of different brightness and compared the input and fitted fluxes. In more than $68 \%$ of the cases, the fitted results are within 0.5 mag of the inputs for

\footnotetext{
22 The doubling time is the time it would take to double the stellar mass of the galaxy, where we have assumed that $50 \%$ of the stellar mass formed is returned to the interstellar medium via supernovae and stellar winds.
} 


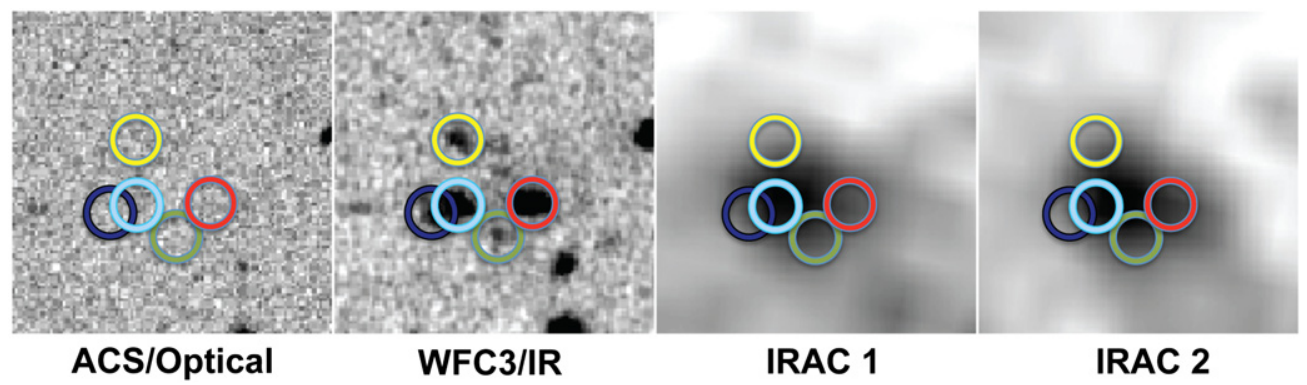

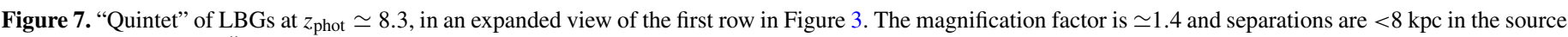

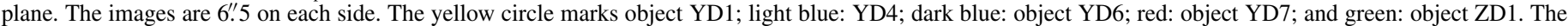

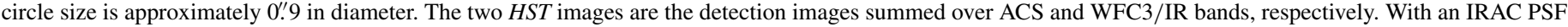

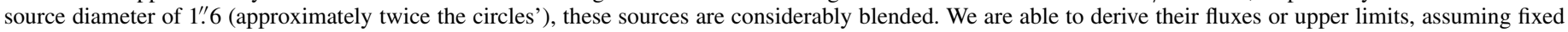
source positions in GALFIT fitting.

(A color version of this figure is available in the online journal.)

three brighter sources in the F160W band, while for the faintest source the output flux decreases with the F160W-IRAC color. Due to the source confusion, our simulations suggest that the de-blending with GALFIT works better for the brighter objects, while it is biased to fainter objects in the sense that their fluxes are likely to be overestimated. This is because that GALFIT often crashes under a very faint flux level. We therefore conclude that we are able to deblend these sources with reasonable accuracy, except for the faint source ZD1 with large uncertainties.

Overdensities in the high-redshift domain have been previously reported. For example, Trenti et al. (2012) identified four candidates with $z \simeq 8$ within 70" in the Field BoRG58. Bradley et al. (2012) found seven LBGs at $z \simeq 7$ in the WFC3/IR field $\left(\sim 120^{\prime \prime} \times 130^{\prime \prime}\right)$ of A1703. However, our finding of nine LBGs at $7<z<9$ within $20^{\prime \prime}(\sim 80 \mathrm{kpc}$ in the source plane) is unique, suggesting that the cosmic variance in source density (Trenti \& Stiavelli 2008) is more significant than anticipated. It appears that cosmic variance increases with redshift (Moster et al. 2011; Bouwens et al. 2014b), as evidenced by different results from surveys. This trend might explain both the large number of clustered $z \simeq 8$ sources and the deficiency of $z \gtrsim 9$ sources and illustrate the need for observations in more fields.

\section{CONCLUSION}

We find 24 LBG candidates at $7 \lesssim z \lesssim 10.5$ in the HFF imaging of A2744, reaching an intrinsic magnitude of $\sim 32$. One source at $z \simeq 7.4$ is lensed into three images. Significant clustering is observed on the intrinsic scale of 10-100 kpc. Thanks to gravitational lensing, we are able to carry out Spitzer/ IRAC photometry for 16 of the sources. SED fitting to the brightest candidates suggests stellar masses of $\simeq 10^{9} M_{\odot}$, star formation rates of $\simeq 4 M_{\odot}$ per year, and a typical formation redshift of $z \simeq 19$.

The redshift distribution of our sample is not a smoothly declining function towards higher redshift. In particular, our redshift distribution does not extend smoothly beyond $z \simeq 9$, clustering notwithstanding, Considering the effect of cosmic variance, the number density in our sample is consistent with that derived from other studies, e.g., Bouwens et al. (2014b). Given the level of clustering that we see in A2744, it will be important to average over more HFF, and to perform a luminosity function analysis so that the redshift dependence can be better related to galaxy mass.

The work presented in this paper is based on observations made with the NASA/ESA Hubble Space Telescope, and has been supported by award AR-13279 from the Space Telescope Science Institute (STScI), which is operated by the Association of Universities for Research in Astronomy, Inc. under NASA contract NAS 5-26555. It is also based on data obtained with the Spitzer Space Telescope, which is operated by the Jet Propulsion Laboratory, California Institute of Technology under a contract with NASA. This work utilizes gravitational lensing models produced by PIs Bradac, Ebeling, Merten \& Zitrin, Sharon, and Williams, funded as part of the HST Frontier Fields program conducted by STScI. These models were calibrated using arcs identified in archival HST imaging by Merten et al. (2011), spectroscopic redshifts of arcs obtained using the VLT/FORS2 spectrograph (Richard et al. 2014), and VLT and Subaru/Suprimecam imaging of the A2744 field (Cypriano et al. 2004; Okabe \& Umetsu 2008; Okabe et al. 2010a, 2010b). X.S. acknowledges support from FP7-SPACE-2012-ASTRODEEP-312725, and NSFC grants 11103017 and 11233002 . Support for A.Z. is provided by NASA through Hubble Fellowship grant HST-HF-51334.01-A awarded by STScI. F.E.B. acknowledges support from BasalCATA PFB-06/2007, CONICYT-Chile grants (FONDECYT 1141218, ALMA-CONICYT 31100004, Gemini-CONICYT 32120003, Anillo ACT1101), and the Millennium Institute of Astrophysics (Project IC120009). J.M.D. acknowledges support from the Spanish consolider project CAD2010-00064 and AYA2012-39475-C02-01. We thank M. Meneghitti for helpful comments.

\section{REFERENCES}

Atek, H., Richard, J., Kneib, J.-P., et al. 2014, ApJ, 786, 60 (A14) Beckwith, S. V. W., Stiavelli, M., Koekemoer, A. M., et al. 2006, AJ, 132, 1729 Benítez, N. 2000, ApJ, 536, 571

Bertin, E., \& Arnouts, S. 1996, A\&AS, 117, 393

Blakeslee, J. P., Anderson, K. R., Meurer, G. R., Benítez, N., \& Magee, D. 2003, in ASP Conf. Ser. 295, Astronomical Data Analysis Software and Systems XII, ed. H. E. Payne, R. I. Jedrzejewski, \& R. N. Hook (San Francisco, CA: ASP), 257

Bouwens, R. J., Bradley, L. D., Zitrin, A., et al. 2014a, ApJ, in press (arXiv:1211.2230)

Bouwens, R. J., Illingworth, G. D., Labbe, I., et al. 2011, Natur, 469, 504

Bouwens, R. J., Illingworth, G. D., Oesch, P. A., et al. 2010, ApJL, 708, L69

Bouwens, R. J., Illingworth, G. D., Oesch, P. A., et al. 2012, ApJ, 737, 90

Bouwens, R. J., Illingworth, G. D., Oesch, P. A., et al. 2014b, arXiv:1403.4295

Bradley, L. D., Bouwens, R. J., Ford, H. C., et al. 2008, ApJ, 678, 647

Bradley, L. D., Bouwens, R. J., Zitrin, A., et al. 2012, ApJ, 747, 3

Bradley, L. D., Zitrin, A., Coe, D., et al. 2014, ApJ, 792, 76

Chabrier, G. 2003, PASP, 115, 763

Charlot, S., \& Fall, S. M. 2000, ApJ, 539, 718

Coe, D., Benítez, N., Sánchez, S. F., et al. 2006, ApJS, 132, 926 
Coe, D., Zitrin, A., Carrasco, M., et al. 2013, ApJ, 762, 32

Coe, D., Bradley, L., \& Zitrin, A. 2014, arXiv:1405.0011

Conroy, C., \& Gunn, J. E. 2010, ApJ, 712, 833

Conroy, C., Gunn, J. E., \& White, M. 2009, ApJ, 699, 486

Cypriano, E. S., Sodré, L., Jr., Kneib, J.-P., \& Campusano, L. E. 2008, ApJ, 613, 95

Ellis, R. S., McLure, R. J., Dunlop, J. S., et al. 2013, ApJL, 763, L7

Fazio, G. G., Hora, J. L., Allen, L. E., et al. 2004, ApJS, 154, 10

Ferguson, H. C., \& McGaugh, S. S. 1995, ApJ, 440, 470

Fioc, M., \& Rocca-Volmerange, B. 1997, A\&A, 326, 950

Ford, H. C., Bartko, F., \& Bely, P. Y. 1998, Proc. SPIE, 3356, 234

Hack, W. J., Dencheva, N., \& Fruchter, A. S. 2013, in ASP Conf. Ser. 475 , Astronomical Data Analysis Software and Systems XXII, ed. D. Freidel (San Francisco, CA: ASP), 49

Illingworth, G. D., Magee, D., Oesch, P. A., et al. 2013, ApJS, 209, 6

Jouvel, S., Høst, O., Lehav, O., et al. 2014, A\&A, 562, A86

Kimble, R. A., MacKenty, J. W., O’Connell, R. W., \& Townsend, J. A. 2008, Proc. SPIE, 7010, 70101E

Koekemoer, A. M., Ellis, R. S., McLure, R. J., et al. 2013, ApJS, 209, 3

Labbé, I., Bouwens, R., Illingworth, G. D., \& Franx, M. 2006, ApJL, 649, L67

Labbé, I, González, V., Bouwens, R. J., et al. 2010, ApJ, 716, 103

Lam, D., Broadhurst, T., Diego, J. M., et al. 2014, arXiv:1406.2702

Laporte, N., Streblyanska, A., Clement, B., et al. 2014, A\&A, 562, 8

Makovoz, D., \& Khan, I. 2005, in ASP Conf. Ser. 347, Astronomical Data Analysis Software and Systems VI, ed. P. L. Shopbell, M. C. Britton, \& R. Ebert (San Francisco, CA: ASP), 81

Merten, J., Coe, D., Dupke, R., et al. 2011, MNRAS, 417, 333

Moustakas, J., Coil, A., Aird, J., et al. 2013, ApJ, 767, 50

Moster, B. P., Somerville, R. S., Newman, J. A., \& Hans-Walter, R. 2011, ApJ, 731,113
Navarro, J. F., Frenk, C. S., \& White, S. D. M. 1996, ApJ, 462, 563

Oesch, P. A., Bouwens, R. J., Illingworth, G. D., et al. 2010, ApJL, 709, L16

Oesch, P. A., Bouwens, R. J., Illingworth, G. D., et al. 2013, ApJ, 773, 75

Okabe, N., Okura, Y., \& Futamase, T. 2010a, ApJ, 713, 291

Okabe, N., Takada, M., Umetsu, K., et al. 2010b, PASJ, 62, 811

Okabe, N., \& Umetsu, K. 2008, PASJ, 60, 345

Overzier, R. A., Shu, X., Zheng, W., et al. 2009, ApJ, 704, 548

Papovich, C., Finkelstain, S. L., Ferguson, H. C., Lotz, J. M., \& Giavalisco, M. 2011, MNRAS, 412, 1123

Peng, C. Y., Ho, L. C., Impey, C. D., \& Rix, H.-W. 2010, AJ, 139, 2097

Postman, M., Coe, D., Benítez, N., et al. 2012, ApJS, 199, 25

Redlich, M., Bartemann, M., Waizmann, J.-C., \& Fedeli, C. 2012, A\&A, 547,66

Richard, J., Jauzac, M., Limousin, M., et al. 2014, MNRAS, 444, 268

Richard, J., Kneib, J.-P., Ebeling, H., et al. 2011, MNRAS, 414, L31

Sanchez-Blázquez, P., Peletier, R. F., Jiménez-Vicente, J., et al. 2006, MNRAS, 371, 703

Torri, E., Meneghetti, M., Bartelmann, M., et al. 2004, MNRAS, 349,476

Trenti, M., Bradley, L. D., Stiavelli, M., et al. 2012, ApJ, 746, 55

Trenti, M., \& Stiavelli, M. 2008, ApJ, 676, 767

Wuyts, S., Labbé, I., Schreiber, N., et al. 2008, ApJ, 682, 985

Zheng, W., Bradley, L. D., Saraff, A., et al. 2012a, in Seventh Conference on Astronomical Data Analysis, 17 http://ada7.cosmostat.org/proceedings.php Zheng, W., Postman, M., Zitrin, A., et al. 2012b, Natur, 489, 406

Zitrin, A., Broadhurst, T., Umetsu, K., et al. 2009, MNRAS, 396, 1895

Zitrin, A., Menanteau, F., Hughs, J. P., et al. 2013a, ApJL, 770, L15

Zitrin, A., Meneghetti, M., Umetsu, K., et al. 2013b, ApJ, 762, 30

Zitrin, A., Zheng, W., Broadhurst, T., et al. 2014, ApJL, 793, L12 